\title{
RRM1 and ERCC1 as biomarkers in patients with locally advanced and metastatic malignant pleural mesothelioma treated with continuous infusion of low-dose gemcitabine plus cisplatin
}

Wendy Muñoz-Montaño ${ }^{1}$, Sae Muñiz-Hernández ${ }^{2 *}$, Alejandro Avilés-Salas ${ }^{3}$, Rodrigo Catalán ${ }^{1,2}$, Luis Lara-Mejía ${ }^{1}$, Suraj Samtani-Bassarmal ${ }^{4}$, Andres F. Cardona ${ }^{5,6}$, Jorge Mendoza-Desión ${ }^{2}$, Daniel Hernández-Cueto ${ }^{7}$, Altagracia Maldonado ${ }^{7}$, Guillermina Baay-Guzmán 7 , Sara Huerta-Yepes ${ }^{7}$ and Oscar Arrieta ${ }^{1,2^{*}}$ (D)

\footnotetext{
Abstract

Background: Malignant Pleural Mesothelioma (MPM) is a rare but aggressive neoplasia that usually presents at advanced stages. Even though some advances have been achieved in the management of patients with MPM, this malignancy continuous to impose a deleterious prognosis for affected patients (12-18 months as median survival, and 5-10\% 5-year survival rate), accordingly, the recognition of biomarkers that allow us to select the most appropriate therapy are necessary.

Methods: Immunohistochemistry semi-quantitative analysis was performed to evaluate four different biomarkers (ERCC1, RRM1, RRM2, and hENT-1) with the intent to explore if any of them was useful to predict response to treatment with continuous infusion gemcitabine plus cisplatin. Tissue biopsies from patients with locally advanced or metastatic MPM were analyzed to quantitatively asses the aforementioned biomarkers. Every included patient received treatment with low-dose gemcitabine $\left(250 \mathrm{mg} / \mathrm{m}^{2}\right)$ in a 6 -h continuous infusion plus cisplatin $35 \mathrm{mg} / \mathrm{m}^{2}$ on days 1 and 8 every 3 weeks as first-line therapy.

\footnotetext{
*Correspondence: sayide@hotmail.com; ogarrieta@gmail.com; ogar@unam.mx

2Laboratory of Personalized Medicine, Instituto Nacional de Cancerología, San Fernando 22 Sección XVI, Tlalpan, 14080 Mexico City, Mexico

${ }^{1}$ Thoracic Oncology Unit, Instituto Nacional de Cancerología, San Fernando 22 Sección XVI, Tlalpan, 14080 Mexico City, Mexico

Full list of author information is available at the end of the article
}

(c) The Author(s). 2021 Open Access This article is licensed under a Creative Commons Attribution 4.0 International License, which permits use, sharing, adaptation, distribution and reproduction in any medium or format, as long as you give appropriate credit to the original author(s) and the source, provide a link to the Creative Commons licence, and indicate if changes were made. The images or other third party material in this article are included in the article's Creative Commons licence, unless indicated otherwise in a credit line to the material. If material is not included in the article's Creative Commons licence and your intended use is not permitted by statutory regulation or exceeds the permitted use, you will need to obtain permission directly from the copyright holder. To view a copy of this licence, visit http://creativecommons.org/licenses/by/4.0/. The Creative Commons Public Domain Dedication waiver (http://creativecommons.org/publicdomain/zero/1.0/) applies to the data made available in this article, unless otherwise stated in a credit line to the data. 


\begin{abstract}
(Continued from previous page)
Results: From the 70 eligible patients, the mean and standard deviation (SD) for ERCC1, RRM1, RRM2 and hENT-1

were 286,178.3 ( \pm 219, 019.8); 104,647.1 ( $\pm 65,773.4) ; 4536.5$ ( \pm 5, 521.3); and 2458.7 ( \pm 4, 983.4), respectively.

Patients with high expression of RRM1 had an increased median PFS compared with those with lower expression

(9.5 vs 4.8 months, $p=<0.001$ ). Furthermore, high expression of RRM1 and ERCC1 were associated with an

increased median OS compared with their lower expression counterparts; [(23.1 vs 7.2 months for RRM1 $p=<0.001$ )

and $(17.4$ vs 9.8 months for ERCC1 $p=0.018)]$.

Conclusions: ERCC1 and RRM1 are useful biomarkers that predict better survival outcomes in patients with advanced MPM treated with continuous infusion of gemcitabine plus cisplatin.
\end{abstract}

Keywords: RRM1, ERCC1, Immunohistochemistry, Biomarkers, Mesothelioma

\section{Background}

Malignant pleural mesothelioma (MPM) is rare neoplasia highly correlated with asbestos and erionite exposure [1]. Even though occupational exposure to asbestos is progressively decreasing, the incidence and mortality due to MPM are still increasing [1]. According to the World Health Organization (WHO), MPM was responsible for 38,000 deaths worldwide between 1994 and 2008. Interestingly, higher incidence and mortality rates were reported in developed countries such as the United States of America, the United Kingdom, and Japan [2]. In Mexico, MPM is the 34th most frequently diagnosed neoplasia; however, since it is commonly diagnosed in advanced stages, some studies suggest that up to $70 \%$ of cases might remain undiagnosed or unreported [3]. During the last decade, different treatments for MPM have shown some advances; unfortunately, this has not been translated into significantly better outcomes. The combination of pemetrexed plus a platinum agent currently stands as the first-line therapy approved by the US Food and Drug Administration (FDA) to treat advanced MPM. Treatment with this combination is reported to yield 6 months median progression-free survival (PFS), and approximately 15.3 months median overall survival (OS) [4]. In 2014, our group reported a remarkable clinical efficacy using combination chemotherapy with lowdose gemcitabine administered in a six-hour continuous infusion plus cisplatin as first-line therapy for patients with advanced MPM [5].

Great efforts had been made to identify biomarkers that allow us to predict treatment response in patients with MPM; however, currently there is no widespread accepted biomarker or model for predicting therapeutic response in patients with advanced MPM [6-8].

Ribonucleotide reductase is an enzyme responsible for de novo synthesis of most deoxyribonucleotides, the abundance of two subunits of this enzyme, named "Ribonucleotide Reductase M1 \& M2" (RRM1 \& M2), correlate with the ability to repair DNA in cancerous cells [9]. In a similar fashion, Excision repair cross complementation group 1 (ERCC1) performs an essential role in several DNA repair pathways [10]. These molecules (RRM1-M2 and ERCC1) have been proposed as biomarkers to predict treatment response to chemotherapy schemes in patients with several solid tumors [1017]. However, there is insufficient evidence to postulate either of these molecules as an undisputable biomarker for treatment response in patients with MPM. Another molecule named "human equilibrative nucleoside transporter-1" (hENT-1), which is a nucleoside transporter protein that mediates cellular entry of cytotoxic agents used as chemotherapics, had also rose interest as a potential biomarker of response to chemotherapeutic regimens, including those containing gemcitabine [12, 18, 19]. Albeit, its role as a biomarker in MPM has not been studied.

Considering the deleterious prognosis that MPM confers to affected patients and the increasing incidence of this malignancy, identifying biomarkers that aid us in predicting treatment response is a promising area for research. Therefore, our study aimed to analyze if ERCC1, RRM1, RRM2, and hENT-1 correlated with response to low-dose gemcitabine in six-hour continuous infusion plus cisplatin in patients with advanced MPM.

\section{Methods \\ Study design}

Patients with a confirmed diagnosis of advanced MPM that were treated at the Thoracic Oncology Unit of Instituto Nacional de Cancerología (INCan) were enrolled from January 2012 until December 2017 if they fulfilled inclusion criteria. The entire study was performed in accordance with the Declaration of Helsinki, and with the principles of good clinical practice. All patients provided written informed consent to participate, and the study was approved by the scientific and bioethical committees of INCan (010/056/ICI) (CEI/656/10).

Locally advanced or metastatic (clinical stage III-IV, according to the AJCC Cancer Staging Manual, 7th edition) newly diagnosed patients who had never received chemotherapy were eligible. Available tumor sample tissue was necessary to perform biomarkers analyses, 
patients without enough available tissue were excluded. Patients with cardiovascular disease (defined as: coronary artery disease, congestive heart failure or arrhythmia), abnormal renal function (serum creatinine $\geq 1.5 \mathrm{mg} / \mathrm{dL}$ or creatinine clearance $<60 \mathrm{ml} / \mathrm{min} / \mathrm{m}^{2}$ ), abnormal hepatic function (serum bilirubin $\geq 1.6 \mathrm{mg} / \mathrm{dL}$ ) or abnormal bone marrow function (leukocyte $\leq 4,000 / \mu \mathrm{L}$ or platelet count $\leq 100,000 / \mu \mathrm{L}$ ) were excluded.

\section{Samples and data collection}

Tumor samples were obtained from 70 patients with a confirmed diagnosis of MPM. All tissue samples were processed to determine the expression of the RRM1, RRM2, ERCC1, and hENT-1. The clinical and demographic data were extracted from from medical records.

\section{Immunohistochemistry}

The level of expression of ERCC1, RRM1, RRM2, and hENT-1 was determined by immunohistochemistry using the following commercial antibodies: ERCC1 (GTX22356), RRM1 (GTX100758), RRM2 (GTX103193) from Genetex Inc., USA; and hENT (SC-48489) from Santa Cruz Biotechnology, USA. Tissue biopsies were processed to obtain slices of $4 \mu \mathrm{m}$; next, slices were deparaffinized at $60{ }^{\circ} \mathrm{C}$ in dry heat and rehydrated in a series of xylene-ethanol solutions. Then, slices were washed with citrate buffer to increase antigen exposure. Afterward, samples were covered with primary antibody for 2 hours and then washed and incubated with a biotinylated secondary antibody (Universal LSAB kit; Dako Corporation, Carpinteria, CA, USA) for $30 \mathrm{~min}$ at room temperature. Next, streptavidin-horseradish peroxidase conjugate (Universal LSAB kit, Dako Corp) and 3, 3'-diaminobenzidine tetra-hydrochloride (liquid DAB, Dako Corp) were added for $30 \mathrm{~min}$; reaction with diaminobenzidine was stopped by adding distilled water, and the slides were counterstained with Hematoxylin (CatHE-M, Biocare Medical, CA. USA). Finally, slices were mounted in Entellan medium (Merk Co, USA).

\section{Scoring of immunohistochemical staining}

A semi-quantitative assessment of staining was conducted by a senior pathologist, who was blinded to clinical outcomes. Positive expression, indicated by a brown color, was quantified as follows: the total available sample was quantified in each case, only tumor cell regions were considered (if the sample contained inflammatory regions, these regions were excluded from analysis). Available sample was divided virtually into $300 \mu \mathrm{m}^{2} \mathrm{sec}$ tions, to eliminate variations, the summatory of all parts was carried out and the total number of pixels obtained was reported for each sample and each biomarker. The staining density was analyzed using the Image Pro-plus 7.0 software (MediaCybernetics, Rockville, MD, USA), which was obtained with the diffusion of light wavelength through the color density in the cells that stained brown. Both negative and positive (weakly positive, moderately positive, and strongly positive) intensities were assessed in tumoral cells. For staining digital analysis, we took into account cytoplasm and nuclear expression (RRM1, RRM2 and hENT-1 were almost exclusively localized on cytoplasm; while ERCC1 was localized at nucleus exclusively).

\section{Treatment}

All patients received chemotherapy with low dose gemcitabine $250 \mathrm{mg} / \mathrm{m}^{2}$ administered in a six-hours continuous infusion plus cisplatin $35 \mathrm{mg} / \mathrm{m}^{2}$ on days 1 and 8 every 3 weeks for up to 6 cycles, or until disease progression, development of unacceptable toxicity, or

Table 1 General characteristics of patients

\begin{tabular}{|c|c|}
\hline & $\begin{array}{l}\text { Total } \\
\%(n) N=70\end{array}$ \\
\hline \multicolumn{2}{|l|}{ Sex } \\
\hline Male & $64.2(46)$ \\
\hline Female & $35.8(24)$ \\
\hline \multicolumn{2}{|l|}{ Age, years } \\
\hline Mean ( \pm SD) & $60( \pm 11.1)$ \\
\hline$<60$ years & $47.1(33)$ \\
\hline$\geq 60$ years & $52.9(37)$ \\
\hline \multicolumn{2}{|l|}{ Smoking history } \\
\hline Present & $54.3(38)$ \\
\hline Absent & $45.7(32)$ \\
\hline \multicolumn{2}{|c|}{ Asbestos exposure } \\
\hline Present & $51.4(36)$ \\
\hline Absent & $48.6(34)$ \\
\hline \multicolumn{2}{|c|}{ Wood-smoke exposure } \\
\hline Absent & $64.3(45)$ \\
\hline Present & $35.7(25)$ \\
\hline \multicolumn{2}{|l|}{ ECOG-PS } \\
\hline $0-1$ & $71.4(50)$ \\
\hline 2 & $28.6(20)$ \\
\hline \multicolumn{2}{|l|}{ Histology } \\
\hline Epithelial & $82.9(58)$ \\
\hline Other & $17.1(12)$ \\
\hline \multicolumn{2}{|l|}{ Karnofsky } \\
\hline$<80$ & $41.4(29)$ \\
\hline$\geq 80$ & $58.6(41)$ \\
\hline \multicolumn{2}{|l|}{ Stage disease } \\
\hline III & $30.0(21)$ \\
\hline IV & $70.0(49)$ \\
\hline
\end{tabular}


withdrawal of informant consent. Those patients with unacceptable toxicity to cisplatin received carboplatin (AUC 5 every 3 weeks on day 1 of each cycle) without changing gemcitabine dosage.

\section{Survival assessment}

PFS, OS, and ORR were correlated according to the expression of the four biomarkers. PFS was defined as the period from starting treatment to disease progression or death. OS was defined as the time from diagnosis to death or loss of follow-up. The ORR was the sum of complete response and partial response assessed by a CT-scan or PET/CT every three cycles according to the modified Response Evaluation Criteria in Solid Tumors (RECIST) for MPM version 1.0 [20]. Adverse events were graded with the Common Terminology Criteria for Adverse Events, version 4.0.

\section{Statistical analysis}

For descriptive purposes, continuous variables were summarized as arithmetic means and SD; categorical variables were comprised as frequencies and proportions. Patients were categorized into high or low expression group based on the four biomarkers median expression. For survival analysis, all variables were dichotomized. The OS and PFS were analyzed by the Kaplan-Meier method, and comparisons among subgroups were assessed by the log-rank test. Patients who lost follow-up were censored from survival analysis. Finally, we performed a multivariate analysis with a Cox proportional model to estimate the hazard ratios with 95\% CI for disease progression and death. Statistical significance was predetermined to be present for values of $p<0.05$ based on a two-sided test. All statistical analyses were carried out using the SPSS (Statistical Package for the Social Sciences) version 24.0 (SPSS Inc., Chicago, IL).

\section{Results}

\section{Study sample}

From a total of 70 included patients, the median age at diagnosis was 60 years, males represented in $64.2 \%$ of population, prior exposure to asbestos was reported in $51.4 \%$ of patients, smoking history was reported in 54.3\%. Most patients were diagnosed with stage IV MPM (70\%); 71.4\% had an ECOG-PS grade of 0-1. Epithelioid MPM was the most common histological subtype, identified in $82.9 \%$ of patients (Table 1 ).

\section{Expression level of biomarkers}

We obtained tumor sample for biomarker assessment from every patient; however, enough tissue to perform IHC to analyze all biomarkers was not available in every patient (Fig. 1). Patients were classified into high or low

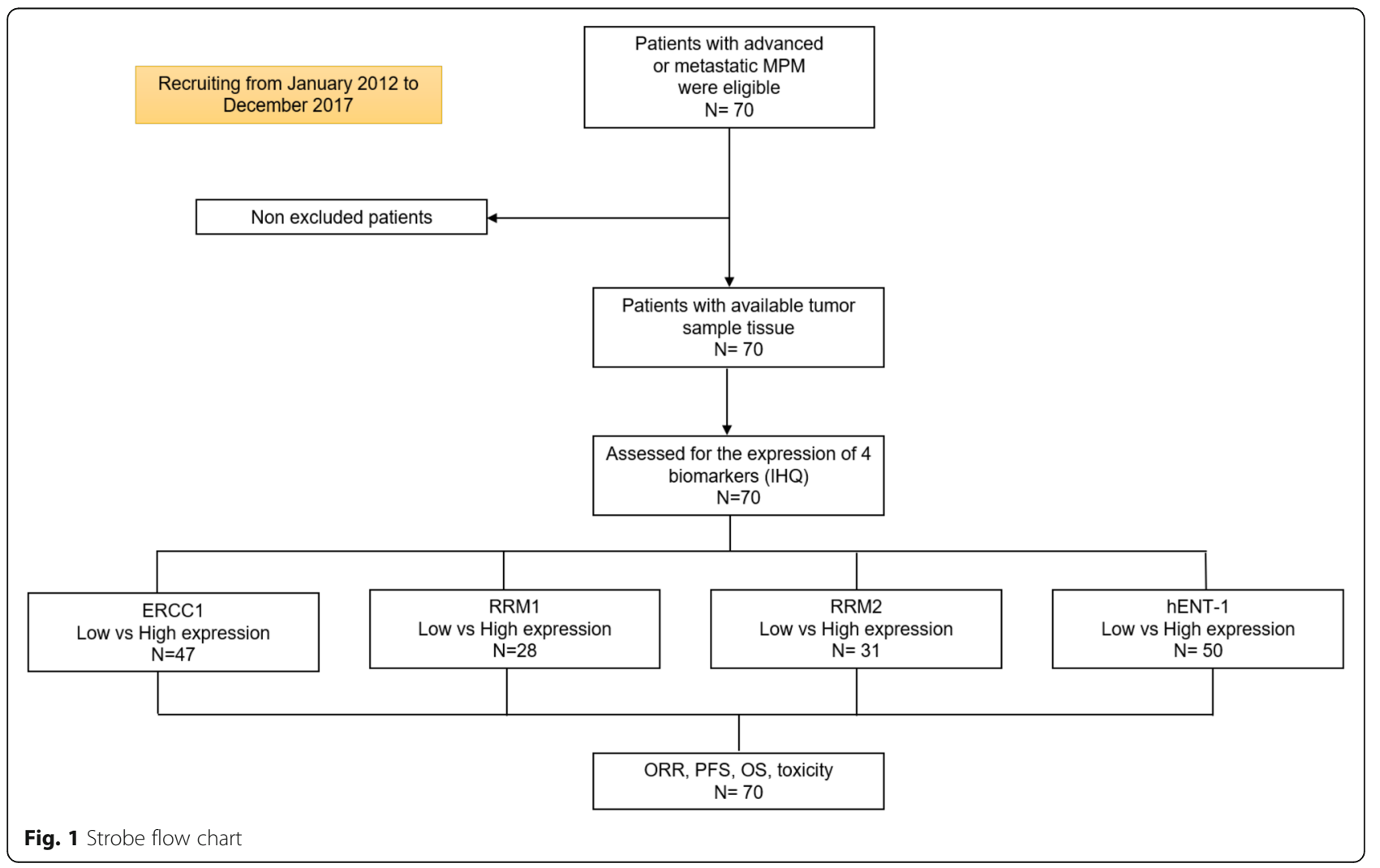


expression groups according to the median value of expression in each biomarker. Figure 2 shows a representative image of each biomarker according to expression level (low or high). The mean and SD for expression of ERCC1, hENT-1, RRM1 and RRM2 was: 286,178.3 ( \pm 219, 019.8); 194,647.1 ( $\pm 104,647.1) ; 4536.5$ ( \pm 5, 521.3) and $2458.7( \pm 4,983.4)$, respectively (Table 2$)$. Distribution was similar between high and low expression groups in all four biomarkers.

\section{ORR, PFS, and OS}

All patients received first-line therapy with low-dose gemcitabine $\left(250 \mathrm{mg} / \mathrm{m}^{2}\right.$ in a 6 -h continuous infusion in days 1 and 8$)$, plus cisplatin $(35 \mathrm{mg} / \mathrm{m} 2$ in day one every 21 days) for a median number of 6 cycles $[1-8,11,12$,
20]. The overall response rate was $47.1 \%(33 / 70)$, and the disease control rate was $80 \%$ (56/70). No complete responses were observed (Table 3).

The median PFS was 7.6 months (4.2-11.1 months). According to the univariate survival analysis, only epithelioid histology subtype was associated with an increased PFS [9.5 months 95\%CI (0.0-19.9 months), $p=$ 0.013]. Biomarker analysis revealed that high expression of RRM1 was correlated with an increased PFS compared with the low expression group [9.5 months vs. 4.8 months; 95\%CI (NR), $p=0.001$ ] (Fig. 3a). On the other hand, ERCC1 expression was not significantly associated with PFS (7.7 months vs. 7.3 months, $p=0.915$ ) (Fig. 3b). At the univariate analysis, no biomarker was associated with an increased in PFS. At the multivariate analysis,

Expression level

\section{High}
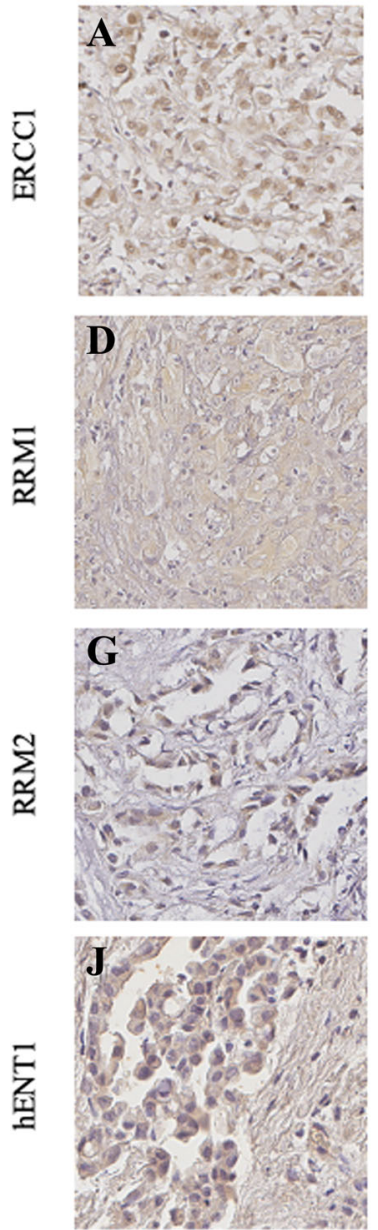

\section{Low}
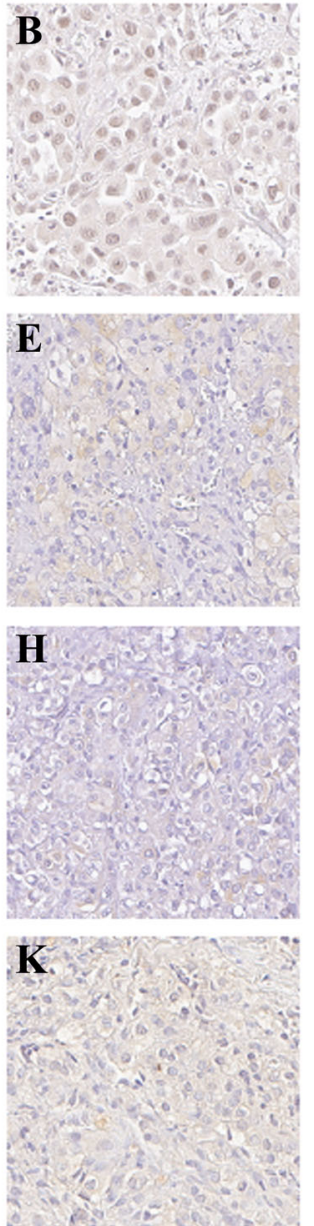

Negative
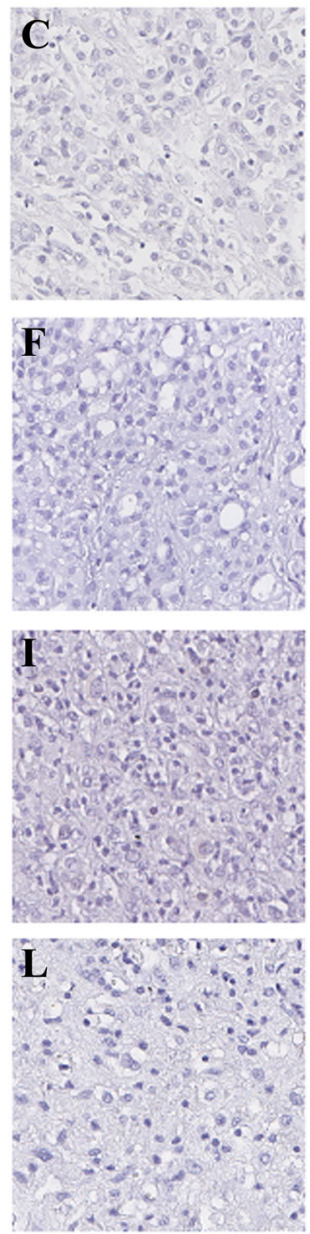

Fig. 2 Immunohistochemical analysis for ERCC1, RRM1, RRM2, and hENT-1 expression in malignant pleural mesothelioma patients. High and low nuclear expression for ERCC1 (a, b), and high and low cytoplasmic expression for RRM1 (d, e), RRM2 (g, h), and hENT-1 (j, $\mathbf{k})$, respectively. Negative controls (without primary antibody) are shown for each molecule in $\mathbf{c}, \mathbf{f}, \mathbf{i}$, and $\mathbf{I}$, respectively 
Table 2 Total expression levels of Biomarkers $(N=70)$

\begin{tabular}{ll}
\hline ERCC1 & $286,178.3( \pm 219,019.8)$ \\
Mean $( \pm$ SD) & $48.9(23)$ \\
Low expression \% (n) & $51.1(24)$ \\
High expression \% (n) & \\
hENT-1 & $104,647.1( \pm 65,773.4)$ \\
Mean ( \pm SD) & $50(25)$ \\
Low expression \% (n) & $50(25)$ \\
High expression \% (n) & \\
RRM1 & $4,536.5( \pm 5,521.3)$ \\
Mean $( \pm$ SD) & $46.4(13)$ \\
Low expression \% (n) & $53.6(15)$ \\
High expression \% (n) & \\
RRM2 & $2458.7( \pm 4,983.4)$ \\
Mean ( \pm SD) & $48.4(15)$ \\
Low expression \% (n) & $51.6(16)$ \\
High expression \% (n)
\end{tabular}

Note: the total of patients varies across the different biomarkers due to the lack of tissue

SD Standard deviation, ERCC1 Excision repair cross complementation-1, hENT-1 Human equilibrative nucleoside transporter-1, RRM1 Ribonucleotide Reductase M1, RRM2 Ribonucleotide Reductase M2

high expression of RRM1 remained significantly associated with a decreased risk of progression [HR 0.11, 95\% CI (0.02-0.52), $P=0.005]$ (Table 4).

\section{OS and clinical features}

The median OS was 14 months (95\% CI 10.5-17.5). At the univariate analysis, high expression of RRM1 and ERCC1 were associated with an increased OS compared with those patients with a low expression; [(17.4 vs 9.8 months; $p=0.018$ ) for RRM1, and (23.1 vs 7.2 months, $p=0.001$ ) for ERCC1] (Fig. $3 \mathrm{C} \& \mathrm{D}$ ). At the multivariate analysis, a Karnofsky Performance Scale Index $>80$, and

Table 3 Characteristics of cisplatin/gemcitabine treatment in the study population

\begin{tabular}{ll}
\hline General data & $\mathbf{N}=\mathbf{7 0}$ \\
\hline Number of cycles & $6(1-11)$ \\
Median (min-max) & $\%(\mathrm{n} / \mathrm{N})$ \\
Overall Response Rate & $47.1(33 / 70)$ \\
Present & $45.7(32 / 70)$ \\
Absent & $7.1(5 / 70)$ \\
Unable to assess & \\
Disease Control Rate & $80(56)$ \\
Yes & $12.9(9)$ \\
No & $7.1(5)$ \\
Unable to assess & \\
\hline
\end{tabular}

high expression of ERCC1, were the only variables significantly associated with a decreased risk of death [HR $0.16,95 \%$ CI (0.04-0.75), $p=0.019$ and HR 0.24, 95\% CI (0.07-0.79), $p=0.020$, respectively] (Table 5).

\section{Toxicity}

Toxicity was assessed in every patient; frequencies and severity of adverse events (AEs) were graded according to the CTCAE V4.0 and reported in Table 6. The most frequent AEs were fatigue, nausea, and vomiting, occurring in $75.7,74.3$, and $25 \%$ of the patients. The most common grade 3 AEs were neutropenia and nausea in 4 and $3 \%$, respectively. No grade 4 or death-related events were observed.

\section{Discussion}

There are none biomarkers that have consistently demonstrated efficacy as predictors of treatment response in patients with MPM. ERCC1, RRM1, RRM2, and hENT-1 have been proposed as potential predictive biomarkers of treatment response in several types of tumors, including lung, pancreatic, ovarian, and colorectal cancer [1016, 18]. Albeit, none of these biomarkers have been widely accepted to be useful in patients with MPM. This study aimed to correlate the expression of four biomarkers with survival outcomes in patients with unresectable MPM treated with low dose continuous infusion of gemcitabine plus cisplatin.

\section{RRM1 and RRM2}

Ribonucleotide Reductase (RR) is a multimeric enzyme responsible for ribonucleoside diphosphate conversion to deoxyribonucleoside diphosphates. This substrate of the DNA polymerases is responsible for the synthesis of de novo deoxynucleotides. RR consists of two subunits; the large subunit M1 (RRM1), which is the regulatory one, and the small subunit M2 (RRM2), which has catalytic functions; combined, these two subunits form the active enzyme [9]. Some therapeutic agents, such as gemcitabine, are directed toward inhibiting the activity of RR. Accordingly, several trials have studied the role of RRM1 expression and the response of patients under treatment with gemcitabine.

Moreover, RRM1 has been reported as a biomarker of treatment response in several neoplasms, such as MPM, non-small cell lung cancer and pancreatic adenocarcinoma; however, results from these studies are not conclusive about the role of RRM1 as a biomarker for treatment response in patients with MPM [11, 16, 18]. Furthermore, there is evidence suggesting that the utility of RRM1 as a biomarker might depend on the therapeutic regimen employed. Zimling ZG et al. previously reported that a lower expression of RRM1 was associated with a better prognosis [21]. In contrast, our 

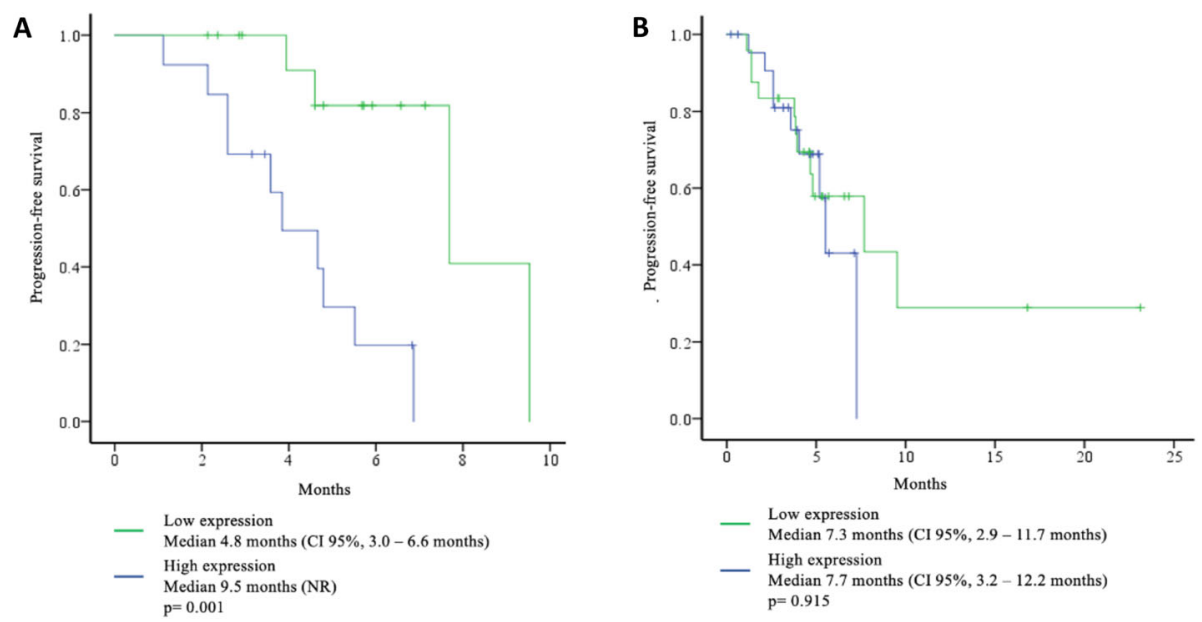

— Low expression

(C1 95\%, $2.9-11.7$ months)

_ High expression Median 7.7 months (Cl 95\%, 3.2 - 12.2 months) $\mathrm{p}=0.001$
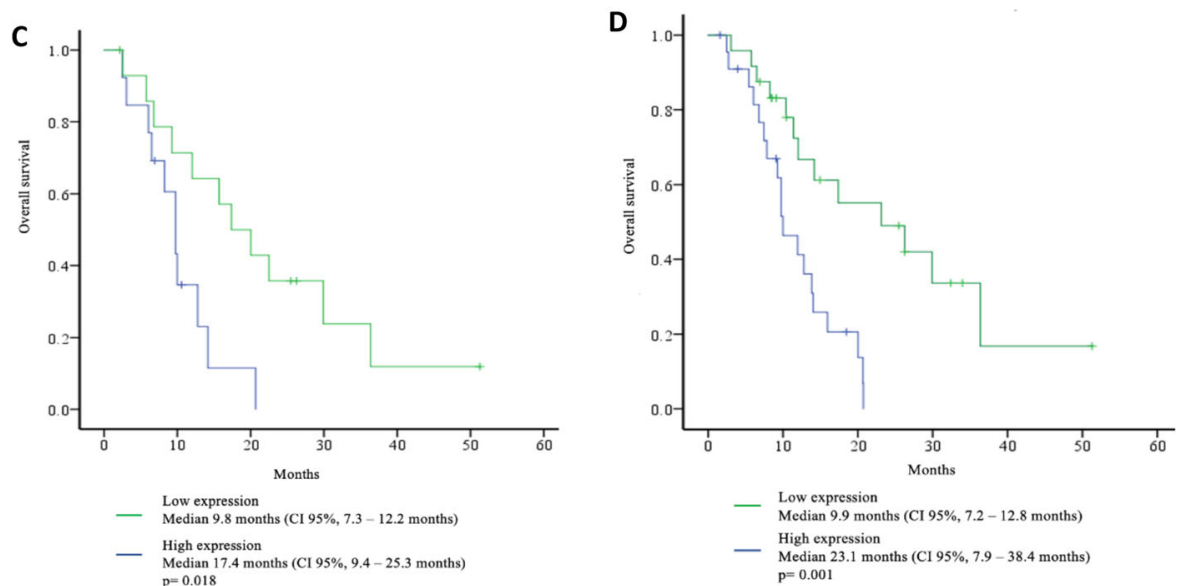

Fig. 3 Progression-free survival (PFS) according to RRM1 (a) and ERCC1 (b) expression, high vs low. Overall survival (OS) according to RRM1 (c) and ERCC1 (d) expression, high vs low

current results suggests that higher expression of RRM1 is associated with increased PFS and OS, being almost twice as high in the group with high expression compared with the group that expressed lower levels of RRM1. These results discrepancies could be explained due to the therapeutic regimen used in each study. Patients enrolled at Zimling ZG et al. study received either pemetrexed-platinum doublet or vinorelbine-platinum doublet, and only patients that presented a high expression of RRM1 and received vinorelbine showed better response (47.1\% vs. $13.3 \%$, negative vs. positive) [21]. Recently, Zito Marino et al., reported thar RRM1 and ERCC1 co-expression was significantly related with worse outcomes in patients with MPM [22]. Even though results from both of these studies contrast with those reported in our present study, it should be underscored that chemotherapy schemes were different to the one we employed. This observation strengthens the hypothesis that the prognostic significance of RRM1 might vary according to the employed chemotherapy. Supporting our results, Frischknecht $\mathrm{L}$ et al., reported an increased freedom from recurrence (FFR), and a better OS in patients with MPM and high expression of RRM1 that received platinum-gemcitabine doublet as first line of therapy [23]. Moreover, in 2014 Szulkin et al., reported that the proportion of malignant cells and RRM1 reactivity in the pleural effusions of patients with MPM correlate to drug sensitivity and survival time, therefore analyzing RRM1 in pleural effusion might be a plausible way for determining which is the best therapy in the first-line setting [24].

\section{ERCC1}

ERCC1 has a cornerstone role in DNA repair pathways; however, similar to what happens with RRM1, the prognostic role of ERCC1 is uncertain considering previous contradictory results. Evidence has correlated negative ERCC1 expression with increased FFR compared with 
Table 4 Unadjusted and adjusted hazard ratios for the factors associated with PFS to cisplatin/gemcitabine among patients with MPM

\begin{tabular}{|c|c|c|c|c|}
\hline & HR $(95 \% \mathrm{Cl})$ & $p$-Value & HR $(95 \% \mathrm{Cl})$ & $p$-value \\
\hline Sex & $1.83(0.85-3.97)$ & 0.124 & $1.36(0.46-4.01)$ & 0.573 \\
\hline Age, $60+$ years & $1.27(0.58-2.76)$ & 0.552 & & \\
\hline Tobacco exposure & $1.08(0.49-2.37)$ & 0.843 & & \\
\hline Exposure to asbestos & $0.61(0.28-1.33)$ & 0.218 & & \\
\hline ECOG PS & $2.01(0.92-4.39)$ & 0.080 & & \\
\hline Histology & $2.90(1.20-7.02)$ & 0.018 & & \\
\hline Karnofsky & $0.61(0.28-1.33)$ & 0.215 & & \\
\hline Disease stage & $0.74(0.34-1.63)$ & 0.457 & & \\
\hline ERCC1 & $0.95(0.39-2.31)$ & 0.915 & & \\
\hline RRM1 & $0.11(0.02-0.52)$ & 0.005 & $0.11(0.02-0.52)$ & 0.005 \\
\hline RRM2 & $0.71(0.24-2.09)$ & 0.532 & & \\
\hline hENT-1 & $1.13(0.47-2.74)$ & 0.780 & & \\
\hline
\end{tabular}

ECOG-PS Eastern Cooperative Oncology Group - Performance Status, ERCC1 Excision repair cross complementation-1, hENT-1 Human equilibrative nucleoside transporter-1, RRM1 Ribonucleotide Reductase M1, RRM2 Ribonucleotide Reductase M2

its positive counterpart in patients with MPM treated with platinum-based chemotherapy [23]. On the same line Zimling ZG et al., reported a significant correlation between negative ERCC1 expression and increased PFS; however, differences were only seen in PFS and did not prevailed when analyzing OS [25]. Two other studies involving MPM patients treated with either pemetrexed as monotherapy, or combined with platinum did not found any association between ERCC1 expression and treatment response [26, 27]. Another trial presented by Cihan et al. studied patients treated with a combination of platinum plus pemetrexed in the first line setting, it was concluded that patients with positive
ERCC1expression presented shorter OS than patients with negative ERCC1 expression (11.7 vs. 19.2 months, respectively). In the same study, a survival analysis at 1 and 2 years reported higher survival rates in patients with no expression of ERCC1 when compared with patients with positive ERCC1 expression (64 and 49\% vs. 40 and $0 \%$, respectively) [28]. Remarkably, we found opposite results, with high ERCC1 expression being marker of increased survival. In our study median OS of patients with high ERCC1 was 23.1 months, considerably higher than its counterpart (7.2 months). One of the main differences between the present study and Cihan and colleagues' work is the therapeutic regimen utilized

Table 5 Unadjusted and adjusted hazard ratios for the factors associated with OS to cisplatin/gemcitabine treatment among patients with MPM

\begin{tabular}{|c|c|c|c|c|}
\hline & HR (95\% Cl) & $p$-Value & HR $(95 \% \mathrm{Cl})$ & $p$-value \\
\hline Sex & $1.21(0.66-2.23)$ & 0.537 & & \\
\hline Age, years & $1.62(0.90-2.95)$ & 0.108 & $2.77(0.75-10.3)$ & 0.125 \\
\hline Tobacco exposure & $0.64(0.35-1.16)$ & 0.141 & $0.82(0.25-2.75)$ & 0.821 \\
\hline Exposure to asbestos & $0.72(0.40-1.31)$ & 0.283 & & \\
\hline ECOG PS & $1.64(0.79-3.43)$ & 0.186 & & \\
\hline Histology & $1.60(0.66-3.84)$ & 0.297 & & \\
\hline Karnofsky & $0.59(0.32-1.11)$ & 0.102 & $0.16(0.04-0.75)$ & 0.019 \\
\hline Disease stage & $0.99(0.53-1.87)$ & 0.979 & & \\
\hline ORR & $2.17(1.25-3.78)$ & 0.006 & $1.55(0.71-3.36)$ & 0.269 \\
\hline ERCC1 & $0.28(0.12-0.63)$ & 0.002 & $0.24(0.07-0.79)$ & 0.020 \\
\hline RRM1 & $0.32(0.12-0.86)$ & 0.023 & & \\
\hline RRM2 & $0.60(0.23-1.41)$ & 0.246 & & \\
\hline hENT-1 & $0.68(0.34-1.39)$ & 0.290 & & \\
\hline
\end{tabular}

ECOG-PS Eastern Cooperative Oncology Group - Performance Status, ERCC1 Excision repair cross complementation-1, hENT-1 Human equilibrative nucleoside transporter-1, RRM1 Ribonucleotide Reductase M1, RRM2 Ribonucleotide Reductase M2 
Table 6 Adverse events $(N=70)$

\begin{tabular}{llllll}
\hline & $\begin{array}{l}\text { Any grade } \\
\%(\mathbf{n})\end{array}$ & $\mathbf{G 1 \%}(\mathbf{n})$ & $\mathbf{G 2 \%}(\mathbf{n})$ & $\mathbf{G 3 \%}(\mathbf{n})$ & $\mathbf{G 4 \%}(\mathbf{n})$ \\
\hline Diarrhea & $21.4(15)$ & $17.1(12)$ & $4.3(3)$ & - & - \\
Nausea & $74.3(52)$ & $35.7(25)$ & $34.3(24)$ & $4.3(3)$ & - \\
Vomit & $35.7(25)$ & $24.3(17)$ & $8.6(6)$ & $2.9(2)$ & - \\
Fatigue & $75.7(53)$ & $40(28)$ & $34.4(24)$ & $1.4(1)$ & - \\
Constipation & $7.1(5)$ & $5.7(4)$ & $1.4(1)$ & - & - \\
Leukopenia & $20(14)$ & $14.3(10)$ & $4.3(3)$ & $1.4(1)$ & - \\
Neutropenia & $20(14)$ & $5.7(4)$ & $8.6(6)$ & $5.7(4)$ & - \\
Neuropathy & $20(14)$ & $11.4(8)$ & $7.1(5)$ & $1.4(1)$ & - \\
Appetite loss & $8.6(6)$ & $4.3(3)$ & $4.3(3)$ & - & - \\
\hline
\end{tabular}

(platinum + pemetrexed vs platinum + gemcitabine). In line with our results, Kao et al., reported that high ERCC1 expression was correlated with longer OS (27.6 vs. 10.3 months; $p=0.06$ ); however, at their study patients underwent surgery (extra pleural pneumectomy) as part of the primary treatment, while in our study surgery was not considered as part of the cornerstone treatment [29].

We consider that there are two major explanations for the discrepancies observed, the first one is that chemotherapy scheme differ among studies; as previously discussed for RRM1, there is a reasonable possibility that ERCC1 perform different according to the therapy employed; another plausible explanation for the discrepancies observed among studies might be the method employed to quantify ERCC1; while we used the median staining value as the cut-off point for determining high vs low expression, most of the aforementioned studies only used positive vs negative expression for stratifying patients. Accordingly, results are inconsistent among studies, with most studies considering high ERCC1 expression as a factor of worse prognosis [22, 30, 31].

\section{hENT-1}

Several studies have reported that hENT-1 expression could be used as a biomarker of treatment response in patients with gastrointestinal cancer who receive treatment with gemcitabine $[12,18,19]$. To our knowledge, the present study is the first to evaluate hENT1 as a biomarker for patients with MPM. Unfortunately, we found no association between hENT-1 expression levels and survival outcomes.

\section{Limitations}

Our study had some limitations, the first one is the relatively small sample size; furthermore, we were unable to obtain enough tissue to evaluate the four proposed biomarkers in the entire population, and this should be considered a significant limitation. Unfortunately, no other study has reported a predefined wll established cut-off value to evaluate the aforementioned biomarkers, further complicating the interpretation of our results and making difficult their comparison with prior studies.

\section{Conclusion}

In patients with advanced MPM that received first line therapy with continuous infusion of low-dose gemcitabine plus cisplatin, the higher expression of RRM1 and ERCC1 are associated with better OS. Furthermore, high expression of RRM1 is also associated with an increased PFS.

\section{Abbreviations}

MPM: Malignant pleural mesothelioma; WHO: World Health Organization; OS: Overall survival; FDA: Food and Drug Administration; PFS: Progression free survival; RRM1 and M2: Ribonucleotide Reductase M1 \& M2; ERCC1: Excision repair cross complementation group 1; hENT-1: human equilibrative nucleoside transporter-1; INCan: Instituto Nacional de Cancerología; ORR: Objective response rate; RECIST: Response Evaluation Criteria in Solid Tumors; EORTC: European Organization for Research and Treatment of Cancer; CALGB: Cancer and Leukemia Group B

\section{Acknowledgements}

Rodrigo Catalán is a doctoral student from Programa de Doctorado en Ciencias Biomédicas at Universidad Nacional Autónoma de México (UNAM) and has received a fellowship (944845) from Consejo Nacional de Ciencia y Tecnologia (CONACYT).

\section{Authors' contributions}

Conception and design: All authors. Administrative support: MMW, MHS, AAS, CR, LML, SBS, OA. Provision of study materials or patients: OA, AAS, MHS, MA, BGG, HYS. Collection and assembly of data: OA, MMW, MHS, CR, LML, CAF. Data analysis and interpretation: OA, MMW, MHS, CR, LML, HCD, MA, BGG, MDJ, HYS. Manuscript writing: All authors. Final approval of manuscript: All authors

\section{Funding}

This work was partially funded by a grant to $\mathrm{SMH}$, by Consejo Nacional de Ciencia y Tecnologia (CONACyT), with registration number 262341; and partially funded by Instituto Nacional de Cancerología (INCan). The funding bodies played no role in the design of the study, collection, analysis of data or in writing the manuscript.

Availability of data and materials

Data generated from this study is available through a reasonable request to corresponding authors.

\section{Declarations}

\section{Ethics approval and consent to participate}

The authors are accountable for all aspects of the work in ensuring that questions related to the accuracy or integrity of any part of the work are appropriately investigated and resolved. The study was conducted in accordance with the Declaration of Helsinki (as revised in 2013). The study was approved by institutional/regional/national ethics/committee/ethics board of INCan [(010/056/ICI) (CEI/656/10)] the and individual consent for this retrospective analysis was waived.

Consent for publication

Not applicable.

Competing interests

All authors have completed the ICMJE uniform disclosure form. 
Dr. Oscar Arrieta has received honoraria as an advisor, participated in speakers' bureau, and given expert opinions to Pfizer, AstraZeneca, Boehringer-Ingelheim, Roche, Lilly, and Bristol-Myers Squibb. The other authors have no conflicts of interest to declare.

\section{Author details}

${ }^{1}$ Thoracic Oncology Unit, Instituto Nacional de Cancerología, San Fernando 22 Sección XVI, Tlalpan, 14080 Mexico City, Mexico. ${ }^{2}$ Laboratory of Personalized Medicine, Instituto Nacional de Cancerología, San Fernando 22 Sección XVI, Tlalpan, 14080 Mexico City, Mexico. ${ }^{3}$ Department of Pathology, Instituto Nacional de Cancerología, Mexico City, Mexico. ${ }^{4}$ Department of Oncology, Bradford Hill, Santiago, Chile. ${ }^{5}$ Foundation for Clinical and Applied Cancer Research (FICMAC), Bogotá, Colombia. ${ }^{6} \mathrm{Clinical}$ and Translational Oncology Group, Clínica del Country, Bogotá, Colombia. ${ }^{7}$ Molecular Markers Laboratory, Hospital Infantil de México Federico Gómez, Secretaría de Salud, Mexico City, Mexico.

Received: 2 November 2020 Accepted: 4 May 2021

Published online: 05 August 2021

\section{References}

1. Tsao AS, Lindwasser OW, Adjei AA, Adusumilli PS, Beyers ML, Blumenthal $\mathrm{GM}$, et al. Current and future management of malignant mesothelioma: a consensus report from the national cancer institute thoracic malignancy steering committee, international association for the study of lung cancer, and mesothelioma applied research foundation. J Thorac Oncol. 2018; 13(11):1655-67. https://doi.org/10.1016/j.jtho.2018.08.2036.

2. Delgermaa V, Takahashi K, Park EK, Le GV, Hara T, Sorahan T. Global mesothelioma deaths reported to the world health organization between 1994 and 2008. Bull World Health Organ. 2011;89(10):716-24, 24A-24C. https://doi.org/10.2471/BLT.11.086678.

3. Aguilar-Madrid G, Robles-Perez E, Juarez-Perez CA, Alvarado-Cabrero I, RicoMendez FG, Javier KG. Case-control study of pleural mesothelioma in workers with social security in Mexico. Am J Ind Med. 2010;53(3):241-51. https://doi.org/10.1002/ajim.20780.

4. Vogelzang NJ, Rusthoven JJ, Symanowski J, Denham C, Kaukel E, Ruffie P, et al. Phase III study of pemetrexed in combination with cisplatin versus cisplatin alone in patients with malignant pleural mesothelioma. J Clin Oncol. 2003;21(14):2636-44. https://doi.org/10.1200/JCO.2003.11.136.

5. Arrieta O, Lopez-Macias D, Mendoza-Garcia VO, Bacon-Fonseca L, MunozMontano W, Macedo-Perez EO, et al. A phase II trial of prolonged, continuous infusion of low-dose gemcitabine plus cisplatin in patients with advanced malignant pleural mesothelioma. Cancer Chemother Pharmacol. 2014;73(5):975-82. https://doi.org/10.1007/s00280-014-2429-5.

6. Curran D, Sahmoud T, Therasse P, van Meerbeeck J, Postmus PE, Giaccone G. Prognostic factors in patients with pleural mesothelioma: the European organization for research and treatment of cancer experience. J Clin Oncol. 1998;16(1):145-52. https://doi.org/10.1200/JCO.1998.16.1.145.

7. Fennell DA, Parmar A, Shamash J, Evans MT, Sheaff MT, Sylvester R, et al. Statistical validation of the EORTC prognostic model for malignant pleural mesothelioma based on three consecutive phase II trials. J Clin Oncol. 2005; 23(1):184-9. https://doi.org/10.1200/JCO.2005.07.050.

8. Herndon JE, Green MR, Chahinian AP, Corson JM, Suzuki Y, Vogelzang NJ. Factors predictive of survival among 337 patients with mesothelioma treated between 1984 and 1994 by the Cancer and leukemia group B. Chest. 1998;113(3):723-31. https://doi.org/10.1378/chest.113.3.723.

9. Engstrom Y, Eriksson S, Jildevik I, Skog S, Thelander L, Tribukait B. Cell cycledependent expression of mammalian ribonucleotide reductase. Differential regulation of the two subunits. J Biol Chem. 1985;260(16):9114-6. https:// doi.org/10.1016/S0021-9258(17)39337-7.

10. Choueiri MB, Shen JP, Gross AM, Huang JK, Ideker T, Fanta P. ERCC1 and TS expression as prognostic and predictive biomarkers in metastatic Colon Cancer. PLoS One. 2015;10(6):e0126898. https://doi.org/10.1371/journal.pone.0126898.

11. Zhu CM, Lian XY, Bi YH, Hu CC, Liang YW, Li QS. Prognostic value of ribonucleotide reductase subunit M1 (RRM1) in non-small cell lung cancer: a meta-analysis. Clin Chim Acta. 2018;485:67-73. https://doi.org/10.1016/j. cca.2018.05.042.

12. Sierzega M, Pach R, Kulig P, Legutko J, Kulig J. Prognostic implications of expression profiling for gemcitabine-related genes (hENT1, dCK, RRM1, RRM2) in patients with Resectable pancreatic adenocarcinoma receiving adjuvant chemotherapy. Pancreas. 2017:46(5):684-9. https://doi.org/10.1097/ MPA.0000000000000807.

13. Han QL, Zhou YH, Lyu Y, Yan H, Dai GH. Effect of ribonucleotide reductase M1 expression on overall survival in patients with pancreatic cancer receiving gemcitabine chemotherapy: a literature-based meta-analysis. J Clin Pharm Ther. 2018;43(2):163-9. https://doi.org/10.1111/jcpt.12655.

14. Liu X, Zhou B, Xue L, Yen F, Chu P, Un F, et al. Ribonucleotide reductase subunits M2 and p53R2 are potential biomarkers for metastasis of colon cancer. Clin Colorectal Cancer. 2007;6(5):374-81. https://doi.org/10.3816/ CCC.2007.n.007.

15. Jordheim LP, Seve $P$, Tredan $O$, Dumontet $C$. The ribonucleotide reductase large subunit (RRM1) as a predictive factor in patients with cancer. Lancet Oncol. 2011;12(7):693-702. https://doi.org/10.1016/S1470-2045(10)70244-8.

16. Chen $Y$, Huang $Y$, Chen DM, Wu C, Leng QP, Wang WY, et al. RRM1 expression and the clinicopathological characteristics of patients with nonsmall cell lung cancer treated with gemcitabine. Onco Targets Ther. 2018; 11:5579-89. https://doi.org/10.2147/OTT.S162667.

17. Fisher SB, Fisher KE, Patel SH, Lim MG, Kooby DA, El-Rayes BF, et al. Excision repair cross-complementing gene-1, ribonucleotide reductase subunit $\mathrm{M} 1$ ribonucleotide reductase subunit $\mathrm{M} 2$, and human equilibrative nucleoside transporter-1 expression and prognostic value in biliary tract malignancy. Cancer. 2013;119(2):454-62. https://doi.org/10.1002/cncr.27739.

18. Kim R, Tan A, Lai KK, Jiang J, Wang Y, Rybicki LA, et al. Prognostic roles of human equilibrative transporter 1 (hENT-1) and ribonucleoside reductase subunit M1 (RRM1) in resected pancreatic cancer. Cancer. 2011;117(14): 3126-34. https://doi.org/10.1002/cncr.25883.

19. Santini D, Vincenzi B, Fratto ME, Perrone G, Lai R, Catalano V, et al. Prognostic role of human equilibrative transporter 1 (hENT1) in patients with resected gastric cancer. J Cell Physiol. 2010;223(2):384-8. https://doi. org/10.1002/jcp.22045.

20. Byrne MJ, Nowak AK. Modified RECIST criteria for assessment of response in malignant pleural mesothelioma. Ann Oncol. 2004;15(2):257-60. https://doi. org/10.1093/annonc/mdh059.

21. Zimling ZG, Santoni-Rugiu E, Bech C, Sorensen JB. High RRM1 expression is associated with adverse outcome in patients with cisplatin/Vinorelbine-treated malignant pleural mesothelioma. Anticancer Res. 2015;35(12):6731-8.

22. Zito Marino F, Baselice S, Erra S, Ronchi A, Montella M, Morgillo F, et al. Double-staining immunohistochemistry reveals in malignant pleural mesothelioma the Coexpression of ERCC1 and RRM1 as a frequent biological event related to poorer survival. Appl Immunohistochem Mol Morphol. 2020 Aug 24;Publish Ahead of Print. https://doi.org/10.1097/PAl. 0000000000000869 . Epub ahead of print. PMID: 32842027.

23. Frischknecht $L$, Meerang M, Soltermann A, Stahel R, Moch H, Seifert B, et al. Importance of excision repair cross-complementation group 1 and ribonucleotide reductase $\mathrm{M} 1$ as prognostic biomarkers in malignant pleural mesothelioma treated with platinum-based induction chemotherapy followed by surgery. J Thorac Cardiovasc Surg. 2015;149(6):1539-46 e1. https://doi.org/10.1016/j.jtcvs.2015.01.065.

24. Szulkin A, Otvös R, Hillerdal CO, Celep A, Yousef-Fadhel E, Skribek H, et al. Characterization and drug sensitivity profiling of primary malignant mesothelioma cells from pleural effusions. BMC Cancer. 2014;24(14):709. https://doi.org/10.1186/1471-2407-14-709 PMID: 25253633; PMCID: PMC4190467.

25. Zimling ZG, Sorensen JB, Gerds TA, Bech C, Andersen CB, Santoni-Rugiu E. Low ERCC1 expression in malignant pleural mesotheliomas treated with cisplatin and vinorelbine predicts prolonged progression-free survival. J Thorac Oncol. 2012;7(1):249-56. https://doi.org/10.1097/JTO.0b013e318233d6a9.

26. Righi L, Papotti MG, Ceppi P, Bille A, Bacillo E, Molinaro L, et al. Thymidylate synthase but not excision repair cross-complementation group 1 tumor expression predicts outcome in patients with malignant pleural mesothelioma treated with pemetrexed-based chemotherapy. J Clin Oncol. 2010;28(9):1534-9. https://doi.org/10.1200/JCO.2009.25.9275.

27. Zucali PA, Giovannetti E, Destro A, Mencoboni M, Ceresoli GL, Gianoncelli L, et al. Thymidylate synthase and excision repair cross-complementing group1 as predictors of responsiveness in mesothelioma patients treated with pemetrexed/carboplatin. Clin Cancer Res. 2011;17(8):2581-90. https://doi. org/10.1158/1078-0432.CCR-10-2873.

28. Cihan YB, Ozturk A, Arslan A, Deniz K, Baran M, Karaca H. ERCC1 as a biological marker guiding management in malignant pleural mesothelioma. Asian Pac J Cancer Prev. 2014;15(10):4117-23. https://doi.org/10.7314/A PJCP.2014.15.10.4117. 
29. Kao SC, Lee K, Klebe S, Henderson D, McCaughan B, Vardy J, et al. Excision repair cross complementation group 1 and thymidylate synthase expression in patients with mesothelioma. Clin Lung Cancer. 2013 Mar;14(2):164-71. https://doi.org/10.1016/j.cllc.2012.09.003 Epub 2012 Oct 17. PMID: 23085037.

30. Ting $S$, Mairinger FD, Hager T, Welter $\mathrm{S}$, Eberhardt WE, Wohlschlaeger J, et al. ERCC1, MLH1, MSH2, MSH6, and Blll-tubulin: resistance proteins associated with response and outcome to platinum-based chemotherapy in malignant pleural mesothelioma. Clin Lung Cancer. 2013;14(5):558-567.e3. https://doi.org/10.1016/j.cllc.2013.04.013 Epub 2013 Jun 27. PMID: 23810210.

31. Powrózek T, Kowalski DM, Krawczyk P, Ramlau R, Kucharczyk T, KalinkaWarzocha E, et al. Correlation between TS, MTHFR, and ERCC1 gene polymorphisms and the efficacy of platinum in combination with pemetrexed first-line chemotherapy in mesothelioma patients. Clin Lung Cancer. 2014;15(6):455-65. https://doi.org/10.1016/j.cllc.2014.06.009 Epub 2014 Aug 15. PMID: 25246386.

\section{Publisher's Note}

Springer Nature remains neutral with regard to jurisdictional claims in published maps and institutional affiliations.

Ready to submit your research? Choose BMC and benefit from:

- fast, convenient online submission

- thorough peer review by experienced researchers in your field

- rapid publication on acceptance

- support for research data, including large and complex data types

- gold Open Access which fosters wider collaboration and increased citations

- maximum visibility for your research: over $100 \mathrm{M}$ website views per year

At BMC, research is always in progress.

Learn more biomedcentral.com/submissions 